\title{
Complexity and Organized Behaviour within Environmental Bounds (COBWEB): An Agent-Based Approach to Simulating Ecological Adaptation
}

\author{
B. Bass ${ }^{1 *}$ and E. Chan ${ }^{2}$ \\ ${ }^{1}$ Adaptation \& Impacts Research Group, Environment Canada at the University of Toronto, \\ Institute for Environmental Studies, 33 Willcocks Street, Toronto, ON M5S 3E8, Canada \\ ${ }^{2}$ Department of Medicine, Faculty of Health Sciences, McMaster University, 1280 Main Street West Hamilton, ON L8S 4L8, Canada
}

\begin{abstract}
An agent-based simulation model, Complex Organization and Behaviour within Environmental Bounds (COBWEB), has been developed to simulate how a group of agents adapts to environmental variability and change. The environment is an abstraction with properties that are common to many complex systems. The environment is two-dimensional and is characterized by a cellular automaton with multiple resources, variable rates of growth, famines and the presence of immovable objects. The agents are genetic algorithms with modifiable behavioural traits and resource preferences. The agents' strategies are defined by movement, consumption, asexual and sexual reproduction, communication and sharing or stealing resources from other agents. The strategies are determined genetically and through new information acquired from the environment or from other agents. COBWEB has been used to examine the linkages between population growth, resources, the availability of energy and surprise suggesting a role for this software in studying ecological processes, specifically the adaptation of different species to environmental change. The current research results illustrate how COBWEB agents can adapt to variability and change in response to changing resource availability, available energy and crossing threshold values. The second set of experiments indicates the importance of genetic diversity and the ability of an invader to extract more energy from the landscape than the indigenous species. The results illustrate that COBWEB is a useful tool for this sort of research and is capable of generating new insights.
\end{abstract}

Keywords: Complexity, ecological simulation, environmental change, genetic algorithms, invasive species

\section{Introduction}

Environmental systems, be they ecological or socioeconomic, are complex, meaning that they are characterized as exhibiting non-linear behaviours, such as sudden and discontinuous change, multiple attractors, self-organization, chaos, uncertainty and surprise. Complexity results from the interactions between various system components at different scales. The behaviours associated with complex systems are not intuitive and do not conform to a Newtonian description of the linear causality, yet are integral to these systems' capacity to adapt to changes in their external environment. The importance of adaptation has been given prominence by the recent concerns about climate change and it has been highlighted in the Framework Convention on Climate Change and the Kyoto Protocol to that agreement. However, adaptation has always been an important way of coping with change, both in the biological and the human realms. How well a system adapts to change will determine its capacity for long-term survival in the face of environmental variability and change.

Describing the behaviour of complex systems has proven to be a challenge to many disciplines. Understanding and pre-

\footnotetext{
* Corresponding author: brad.bass@ec.gc.ca
}

dicting how a system will adapt to change is a difficult challenge as only some strategies may prove to be successful and the system has to have access to these strategies, either through sufficient biodiversity in ecosystems or through information storage in socio-economic systems. Approaches range from differential equations and iterative function systems for simulation, non-linear regression for empirical description and optimization for decision making. Each of these approaches has its strengths and weaknesses depending on the specific research question or decision under consideration. The approach that is described in this paper is based on simulating the behaviour of individual agents in the environment and it has become known as agent-based simulation, emergent computing or evolutionary computing.

Agent-based simulation is well suited for studying and simulating adaptation. It simulates the choices of each agent in the system, allowing for observation of emergent behaviours at multiple scales, particularly the scale of the individual and at the scale of the whole system. The simulation platform that has been developed for this research, called COBWEB (Complexity and Organized Behaviour Within Environment Bounds), provides a means of conducting experiments on how well a system adapts to both variability and change, at multiple scales, in space and in time. By simulating the decisions of each agent, it is possible to observe the emergence of dominant strategies or species and dynamic spatial patterns as the 
population adapts to variability in the resource base. Environmental change can be represented with specific alterations to energy requirements, energy availability, resource growth rates, inter-agent communication, agent memory, preferences and breeding capabilities. Although COBWEB was not meant to mimic any specific system, it is an abstraction of many complex systems, and as such generates insights that are applicable to a broad range of environmental research questions. This paper illustrates how COBWEB can provide insight into questions involving ecological adaptation to quantitative and qualitative changes in the resource base. A specific example is provided to illustrate how COBWEB can build upon these results to simulate the invasion of one ecosystem by an alien species.

\section{Characterizing Complex Systems}

Complexity emerges out of the interactions between various components of a system. These interactions may lead to multiple attractors, chaotic behaviour and other hallmarks of non-linear behaviour. The existence of multiple attractors allows the system to shift between different states with changes in the inputs of energy, matter or information. Essentially, as the gradient of energy, matter or information across the system changes, the system responds to diffuse the gradient. This requires that the system self-organize into a different structure, thus leading to the emergence of new properties and surprise.

These shifts can occur quite suddenly appearing as discontinuous jumps. Scheffer (1990) demonstrated that in shallow lakes, changing the nutrient input would alter the system, in a discontinuous manner, from a benthic to a pelagic state as the nutrient levels crossed a certain threshold. Sudden change between attractors is common to other natural systems. Severinghaus and Brook (1999) detected that the global climate system experienced 8 sudden changes of mean temperature of at least $10^{\circ} \mathrm{C}$, each occurring in less than a decade. Byers and Hansell (1996) and Bass et al. (1998) have suggested and provide a theoretical basis to suggest the existence of semi-stable attractors. At these attractors, the system appears to be stable but is slowly, almost imperceptibly, shifting to a new state. The shift also appears in a discontinuous manner.

Complex environmental systems, particularly natural systems, are organized around multiple attractors, without a preferred state. For complex environmental systems, the presence of multiple attractors suggests that incremental change in external inputs of matter, energy and information do not result in incremental system change. The changes in external inputs appear to have no effect on the system until a threshold is reached, and then a small change appears to cause a dramatic change of state in the systems. If the system is on a semistable attractor the situation may be somewhat different. Sudden changes may appear without any noticeable change in external inputs. The new attractor may in fact be a preferred state.
Complex systems can be described and understood, but prediction may be difficult due to the presence of multiple attractors. Prediction is further complicated because some of these attractors will be strange, implying chaotic behaviour. Chaos limits how far into the future we can predict. Thus, for example, ten days is considered to be the limit in terms of predicting the weather. Running numerical weather models into the future amplifies small errors, increasing the uncertainty of any prediction. However, it may be possible to predict in a more qualitative manner, by using enough simulations to examine the range of possible futures.

Complex systems also operate with a window of vitality. Too little complexity would not allow the system to adapt to a change in the gradients of energy, matter or information. They must have enough complexity to adjust to changes in these gradients, but too much complexity, on the other hand, would inhibit the system's resilience. The systems strive for an optimum, not a minimum or maximum amount of complexity. This has led some authors to speak in terms of goals, particularly in ecosystems (Müller and Fath, 1998).

\section{Emergent Computing}

Emergent computing is an approach to simulation that seems to best represent the characteristics of complexity as it begins with the interaction between different components of the system. Olson and Sequeira (1995) define an emergent computer system as a system composed of independent agents, which behave according to explicit instructions. The system exhibits implicit spatial and/or temporal patterns that arise as a result of interactions between these sub-components and/or between them and their environment. The patterns are apparent at a higher level than the agents, and are not explicitly coded in their specifications.

The instructions for each agent can be represented using a variety of artificial intelligence tools. One such tool is the genetic algorithm (GA). A GA is string composed of 1's and 0 's that encode how a particular agent will choose from a range of specified behaviours. The output of the agent is in turn used as the input at the next time step in the simulation. As such, the agents function as through a process of feedback. Agents with a set of instructions that is better suited to the environment will be selected to produce offspring. One analogue for a GA is the self-referential sentence. The following example is taken from Hofstadter (1985).

This sentence has how many 0's_, 1's_, 2's_, 3's_, 4's_, 5's_, 6's_, 7's_, 8's_ and 9's_.'.

This sentence has how many 0's 1, 1's 1 , 2's 1 , 3's 1 , 4's 1 , 5's 1 , 6's 1 , 7's 1,8 's 1 and 9's 1 .

Output $t_{n}[1,1,1,1,1,1,1,1,1,1]$

Output $t_{n+1}[1,11,1,1,1,1,1,1,1,1]$

Output $t_{n+2}[1,12,1,1,1,1,1,1,1,1]$

Output $t_{n+3}[1,11,2,1,1,1,1,1,1,1]$

Examples of emergent computation software include 
ECHO, GECKO and Evolve. All of these use genetic algorithms to represent the behaviour of individual agents.

\section{Complexity and Organized Behaviour within Environmental Bounds (COBWEB)}

The COBWEB software platform and variables have been described in Bass et al. (2002). In summary, COBWEB is an object-oriented emergent computing simulation platform implemented in Java to provide flexibility and portability. The platform consists of a two-dimensional multiple resource environment and barriers, referred to as rocks or stones. The resource growth is simulated with a cellular automata algorithm. The grid size is flexible and the user can choose either hard boundaries, an agent hits the boundary is turned around, or wrap around boundaries where an agent hits a boundary and emerges on the opposite side of the grid. The agents are genetic algorithms. The genetic code is randomly assigned at the beginning of a simulation, but changes to the code occur in offspring through mutation and cross over. The initial number of agents is variable and is determined by the user. model:

COBWEB offers many unique features as a simulation

1. It allows for multiple resources, each with its own variability;

2. Environmental change can be introduced suddenly at any point in the program;

3. The agents are classified according to the similarities in their genetic code;

4. It allows for resource preferences amongst the agents;

5. It allows herbivorous, omnivorous, carnivorous or cannibalistic behaviour;

6. Agents can be allowed to store environmental information and communicate with other agents;

7. Agents can be allowed to share or steal energy from other agents;

8. At any point in a simulation, the user can alter the quantity and location of resources, agents and barriers;

9. The agents can exhibit both biological and social behaviours; and

10. The software is both flexible and portable.

Bass et al. (2002) have described how the COBWEB simulations are initiated for an individual run. Briefly, the user is able to adjust several parameters for the environment such as resource growth rates and several energy parameters, such as the amount available through consumption, and the requirements for movement and reproduction. The user is also able to determine how the agents will reproduce, sexually or asexually, the probability of communication and sexual reproduction and the size of the agents' memory and communication buffer, i.e. how much new information the agent can store. The visual display of the program is displayed in Figure 1. The triangles are the agents, the black squares are the barriers and the other squares are the resources. The dots in the triangles indicate the resource preference. Three triangles are not black. They are three genetic markers, coloured red, green and blue. Other agents are given a colour depending on how close they are to any of these three colours.

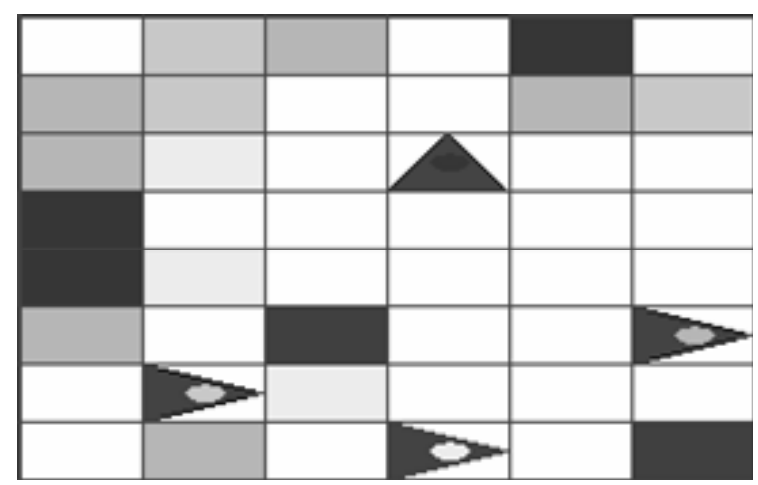

Figure 1. Each triangle is an agent with a randomly assigned genetic algorithm, the dots in the triangle indicate the agent's favourite resource, the dark squares are rocks or solid barriers and the light-coloured squares are food or resources.

\section{General Trends: Variability in Resources}

An analysis of the program's behaviour in response to changes in the resource base was conducted before any specific applications were tested to identify variable-specific thresholds, trends and any irregularities. The following steps were applied to characterize the new COBWEB program:

1. Independent evaluation of the threshold values of each variable, i.e. the bounds within which an equilibrium population can be maintained;

2. Independent evaluation of the population \& resource availability trends within threshold levels of each variable including

- Equilibrium and maximum level of food resources, agent population and average agent energy;

- The time required to reach the maximum agent population and food resources;

3. Preliminary evaluation of altering multiple variables in the same simulation run;

4. Preliminary evaluation of the effects of using different resource preferences amongst agents.

The experiments were conducted with a set of default parameters to establish a baseline for further investigation. The food rate, the rate at which new resources were made available in empty locations independent of existing resources, was set to zero to give the simulation more realism, creating the uneven, patchy resources characteristic of natural habitats. To counterbalance the change in food availability, the initial food resources have been made more plentiful. The initial agent energy and energy cost for breeding have been set low and high respectively, so as to require the parent organism to build up a level of excess resources before committing to 
reproducing and to be able to maintain this level throughout the gestation period. The lower initial energy forces the agent to forage rather than just surviving on its energy stores.

The energy required to move, step energy and turn energy, are low enough to permit the survival of the offspring despite the lower initial energies. However, the energy expended on collisions, step rock energy, is high to represent energy loss due to environmental hazards in natural systems. These values give those agents that demonstrate the ability to avoid the energy loss a survival advantage in the long-term. Other notable characteristics in the default values include:

1. one type of resource;

2. no resource preference amongst agents;

3. no random addition of resources;

4. no communication between agents and no agent memory.

Table 1. Default Parameter Values

\begin{tabular}{ll}
\hline COBWEB Parameters & Default Values \\
\hline Grid Size & 75 by 75 \\
Wrap & True \\
Agent Memory & 0 \\
Communication & 0 \\
Initial Stones & 100 \\
Initial Agents & 100 \\
Initial Food & 3500 \\
Food Rate & 0 \\
Food Grow & 1.5 \\
Mutation Rate & 0.003 \\
Initial Energy & 50 \\
Food Energy & 10 \\
Breed Energy & 100 \\
Asexual Pregnancy Period & 1 \\
Sexual Pregnancy Period & 1 \\
Asexual Breed Chance & 0.25 \\
Sexual Breed Chance & 0.25 \\
Step Energy & 5 \\
Step Rock Energy & 20 \\
Turn Energy & 0 \\
Turn Right Energy & 1 \\
Turn Left Energy & 1 \\
\hline
\end{tabular}

The default values yielded the following results (Figure 2). Clearly, a predator-prey cycling emerges, similar to that noted in Bass and Suh (2002) and Suh (2004) in earlier experiments with COBWEB. The inherent pattern of growth of agents and food remains the same. An initial rapid growth of resources is followed by a rapid growth in the agent population that leads to a sudden decline in resources. The initial decline in resources leads to a decline in the agent population that is followed by a stable fluctuation in both the resources and population, similar to predator-prey cycling. The fluctuations indicate that energy and population are coupled to resource variability, yet the stability of the amplitude over time suggests that the agents have adapted to this baseline level of environmental variability. The even narrower amplitude of the average agent energy indicates that the dominant adaptation strategy is to maintain a level consumption that restricts the variation in the amount of energy for each individual agent.
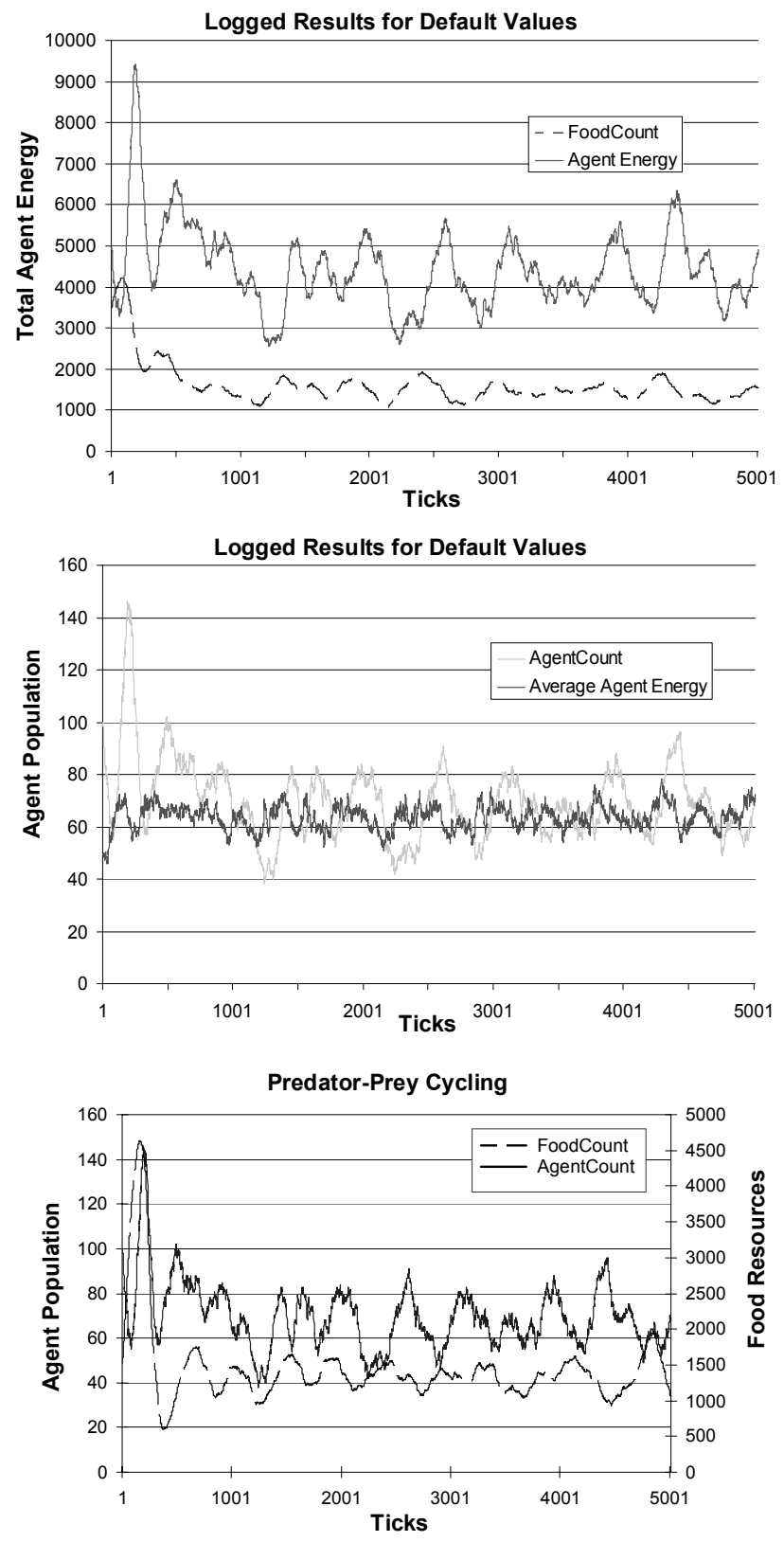

Figure 2. Major trends in simulations conducted with default parameter values.

\section{General Trends: Environmental Change Affecting Resource Availability}

Environmental change is implemented with a multiphase run by modifying a variable that affects resource availability midstream during the simulation, when the amplitudes of the fluctuations in population and resources are relatively stable. Initial runs have been attempted to compare single versus multiphase runs and observe whether there are significant 
Table 2. Comparing Single and Multiphase Runs with changes in the Food Grow Parameter

\begin{tabular}{lllll}
\hline & Food Count & Agent Count & Average Agent Energy & Agent Energy \\
\hline Single Phase, Food Grow = 1.5, Maximum & 4226 & 146 & 78 & 9409 \\
Multi-Phase, Food Grow = 1.5, Maximum & 3961 & 164 & 74 & 9854 \\
Single Phase, Food Grow = 6, Maximum & 5253 & 574 & 83 & 36541 \\
Multi-Phase, Food Grow = 6, Maximum & 4390 & 599 & 93 & 42467 \\
Single Phase, Food Grow = 1.5, Minimum & 1058 & 38 & 46 & 2554 \\
Multi-Phase, Food Grow = 1.5, Minimum & 1434 & 56 & 42 & 3136 \\
Single Phase, Food Grow =6, Minimum & 2097 & 437 & 56 & 26684 \\
Multi-Phase, Food Grow = 6, Minimum & 789 & 466 & 17412 \\
Single Phase, Food Grow = 1.5, Equilibrium & 1452 & 66 & 64 & 4226 \\
Multi-Phase, Food Grow = 1.5, Equilibrium & 1587 & 72 & 51 & 4377 \\
Single Phase, Food Grow =6, Equilibrium & 1128 & 387 & 59 & 22845 \\
Multi-Phase, Food Grow = 6, Equilibrium & 1382 & 416 & 63 & 26008 \\
\hline
\end{tabular}

changes to the manner in which a population adjusts to a changed environment. A multi-phase trial was first attempted on the Food Grow variable to observe the result in a change in the environment on the agent population (See Table 2 and Figure 3). Initially, the variables were set to all default values with food grow at 1.5. After 5000 time steps (an arbitrary number used to ensure that the population is at equilibrium), the food growth rate was increased to 6 . At this greatly increased value, the population increased significantly while food availability remained essentially unchanged after the initial period of adjustment. The total agent energy increased proportionally with the agent population, thus the average agent energy remained relatively unchanged. The second equilibrium was attained quickly with no unexpected fluctuations. The multiphase simulation runs were compared to single phase simulation runs where Food Grow is set to 6 .

It was expected that if the population had adapted to the environment during the initial 5000 time steps, the population should have more difficulty adjusting to the new environmental conditions than the population used in the single-phase runs. The adjusted population would have a lower genetic diversity than the initial population because of the environmental selection pressures. Population adaptation is not measured directly in COBWEB. However, the amplitude of the fluctuations immediately after the shift was taken as an indirect method of quantifying the population adaptability. With changes to the Food Grow parameter, the fluctuations in agent population and energy in the multi-phase run were similar to those seen in the single run reflecting the capacity to adapt to the new environmental settings while maintaining a higher population.

The equilibrium conditions for both the single and multiphase runs approach very similar values providing further evidence for the existence of an 'optimum' level of population, food, and energy for the system with a given long term variability in the environment. In both the single-phase and multi-phase runs, the equilibrium levels of food availability and average agent energy did not vary with changes in the Food Grow parameter indicating that the dominant strategy is to maintain a consistent level of energy for each individual agent.

A similar approach was used to study the effect of famine. After 5000 time steps, a periodic decline of $20 \%$ in resources, every 50 time steps, was introduced to the system. No extreme fluctuations were observed during the shift in environmental conditions, but there were declines in the amounts of food, population and energy. The famine produced similar results in the single phase run except for the food count, which actually increased during the famine when it was implemented at the beginning of the simulation (Table 3). This is most likely due to a rapid decline in the agent population at the beginning of the simulation. Similar to the experiments with increasing amounts of food, the agent population adapted by choosing a strategy that maintained the average energy. After the environmental change, the actual population size decreased, but the average agent energy remained consistent with the level that existed before the change. Thus the consumption strategy that emerged in the baseline is adequate to allow the system to adapt to an increase in food resources, but when resources are decreased, this strategy reduces the total population. Whether this is a successful adaptive strategy would require further experimentation to assess whether the system could have maintained a higher population, over the long term, with reduced consumption and lower energy levels. However, in these restricted conditions, a new agent with a strategy based on lower consumption was not able to emerge to complete with the existing agent strategy due to the low probability of sexual reproduction and the low rate of mutation.

A very different result emerged with changes to Food Energy, the amount of energy available from a single resource, from 10 to 50 (Table 4 and Figure 3). When Food Energy was set to 50 , the single-phase run settled into a steady state after a few hundred time steps, albeit at a much higher level of both average and total energy and a lower population and food count. The higher energy level was not surprising as the energy obtained from consumption was five times higher than in 
Population-Resource Cycling

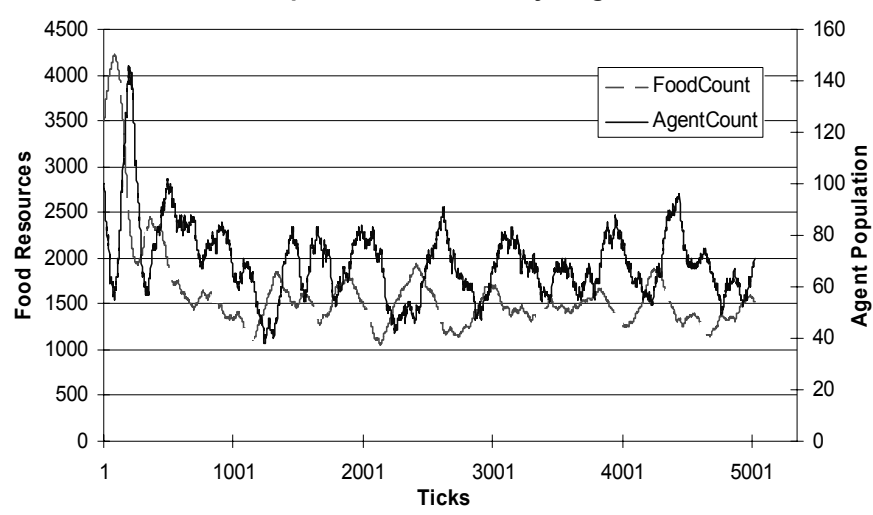

Agent Energy

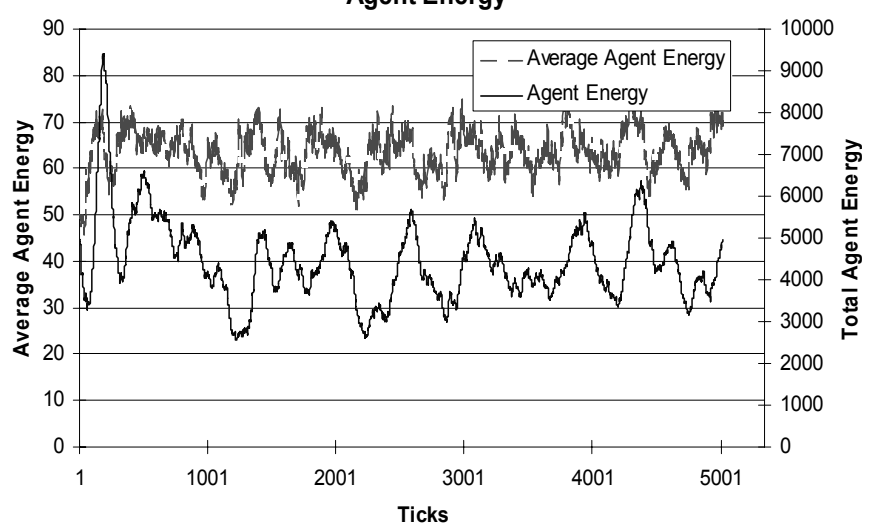

Single Phase Run: Food Grow = 1.5
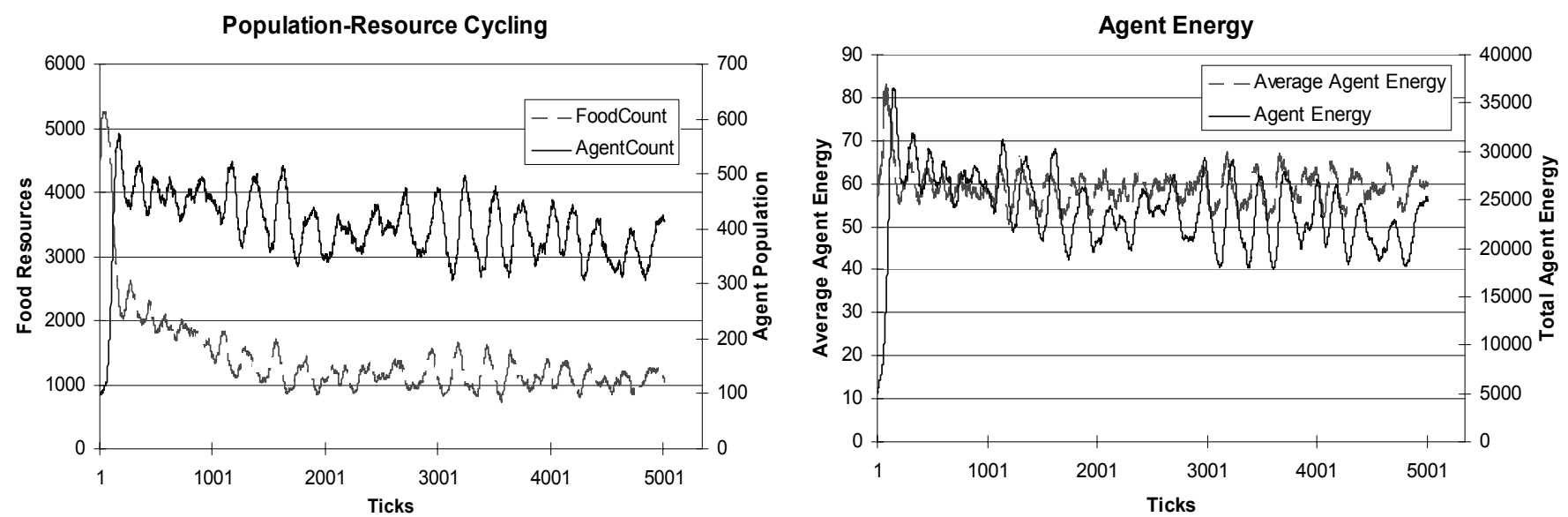

Single Phase Run: Food Grow $=6$

Population-Resource Cycling

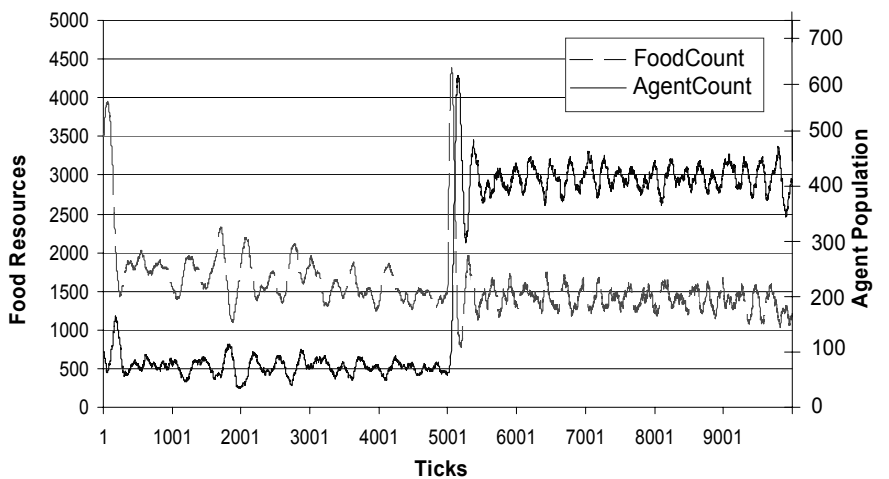

Agent Energy

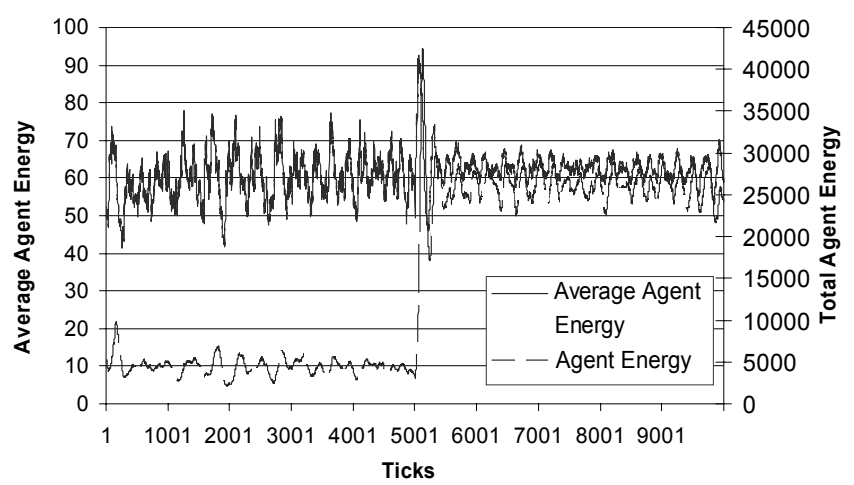

Figure 3. Comparing single and multiphase runs with changes in food grow parameter. 
Table 3. Comparing Single and Multiphase Runs Famine

\begin{tabular}{lllll}
\hline & Food Count & Agent Count & Average Agent Energy & Agent Energy \\
\hline Single-phase Equilibrium, Default & 1452 & 66 & 64 & 4226 \\
Multi-phase, Default Equilibrium & 1739 & 56 & 68 & 3812 \\
Single-phase, Famine Equilibrium & 1645 & 48 & 68 & 3221 \\
Multi-phase, Famine Equilibrium & 1429 & 38 & 68 & 2608 \\
\hline
\end{tabular}

the default run where Food Energy was equal to 10. In the multiphase run, the initial fluctuation in the population after Food Energy was increased was very similar to the single-phase run, but instead of levelling off, the population declined soon after 6000 time steps. The growth in resources was not able to keep up with the explosive growth in agent population. The agents completely consumed the resource base and in turn agent population declined to zero. The maximum values for food and population are very similar for the two runs, but the minimum values for food and population, after the increase in Food Energy, are much lower in the multiphase run.

It is not surprising that an increase in the ability of the agents to use the food resources in their environment would ultimately lead to the failure of the population due to an imbalance of agents and food availability. The food or resources are controlled by a fixed set of rules and do not adapt to the agents. In this case, due to the higher amount of energy available form the food, the consumption increased the average agent energy by an order of magnitude. The additional consumption allowed for more reproduction and a growth in the agent population that led first to the extinction of the food and then to the extinction of the population. Thus the adaptations of the individual agents lead to a system-wide maladaptation.

Interestingly, in the multiphase run, while the total agent energy follows a pattern similar to the agent population, the average agent energy becomes increasingly chaotic in appearance (Figure 3). In particular, the ratio of the variance to the mean is becoming larger. By time step 7000, it is obvious that the surviving agents are successful at finding food and are choosing to maximize their energy as resources become available. This is also seen in Table 4 as the minimum average agent energy is 228, while in the single-phase run it is 61 . With a steady decline in the amount of available food, this level of energy cannot be maintained and is subject to largescale fluctuations. This behaviour in an ecological simulation is characteristic of the occurrence of sudden change at a bifurcation point, in this case representing a failure of the system to adapt to a change in its environment (Hansell et al., 1997). This sudden change in system behaviour also suggests that when Food Energy was raised to 50, it exceeded a threshold value, for the dominant strategy, beyond which the system is not viable given the other environmental and energy parameter values. The presence of threshold values is of significant interest for investigating climate change in the future since it suggests a potential application of COBWEB for elucidating the frequency and characteristics of these unpredictable events.

These experiments indicate that the agents will adapt to changes in the resource base by maintaining the same level of consumption. When the resource base increases or declines,

Table 4. Comparing Single and Multiphase Runs with Changes in the Food Energy Parameter

\begin{tabular}{lllll}
\hline & Food Count & Agent Count & Average Agent Energy & Agent Energy \\
\hline $\begin{array}{l}\text { Single-Phase, Food Energy = 10, Maximum } \\
\text { Multiphase, Food Energy = 10, Maximum }\end{array}$ & 4226 & 146 & 78 & 9409 \\
Single-Phase, Food Energy = 50, Maximum & 4182 & 131 & 76 & 7603 \\
Multiphase, Food Energy = 50, Maximum & 3880 & 597 & 522 & 147840 \\
Single-Phase, Food Energy = 10, Minimum & 3452 & 537 & 510 & 157627 \\
Multiphase, Food Energy = 10, Minimum & 1058 & 38 & 46 & 2554 \\
Single-Phase, Food Energy = 50, Minimum & 1954 & 48 & 44 & 2461 \\
Second Minimum & 124 & 54 & 61 & 2953 \\
Single-Phase, Food Energy = 10, Equilibrium & 1452 & 10 & 228 & 4226 \\
Multiphase, Food Energy = 10, Equilibrium & 2114 & 66 & 64 & 3206 \\
Single-Phase, Food Energy = 50, Equilibrium & 232 & 87 & 49 & 29591 \\
Multiphase, Food Energy = 50, Equilibrium & - & - & 344 & - \\
\hline
\end{tabular}



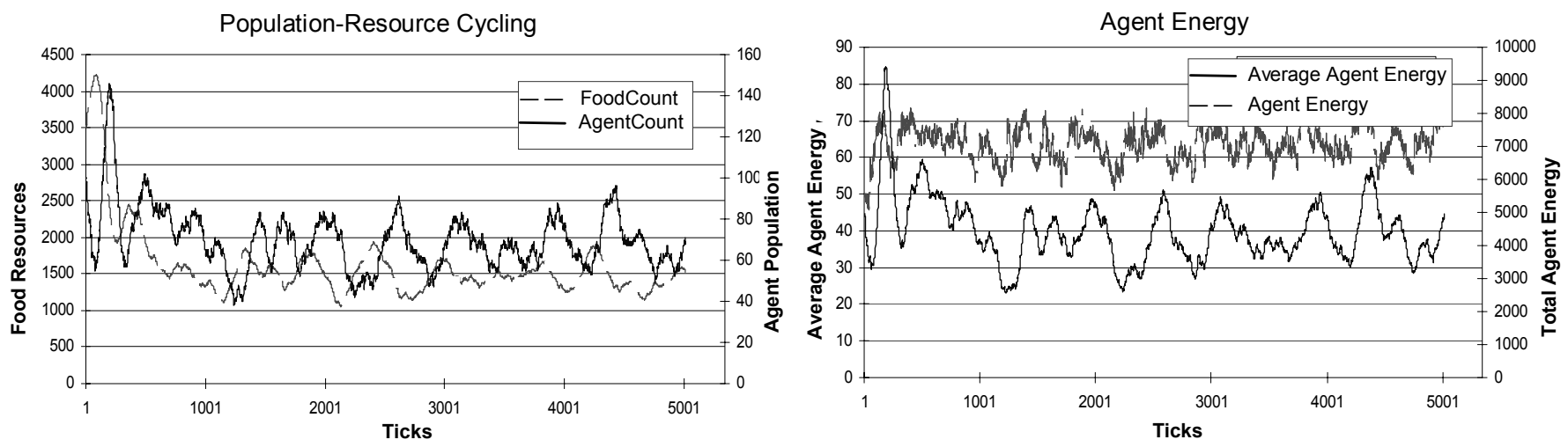

Single Phase Run: Food Energy = 10
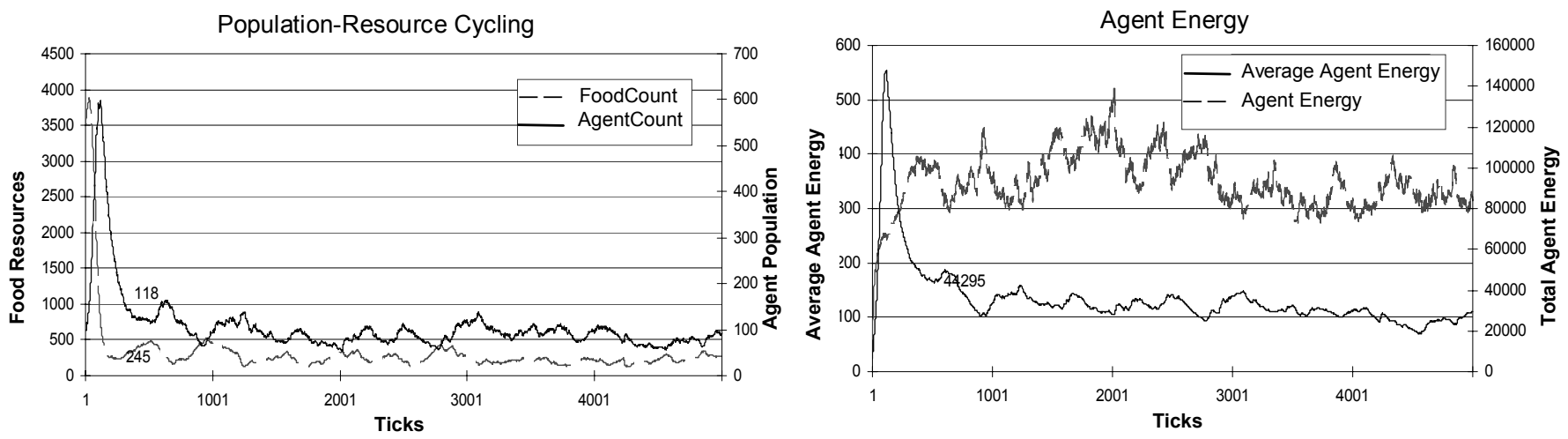

Single-Phase Run: Food Energy $=50$
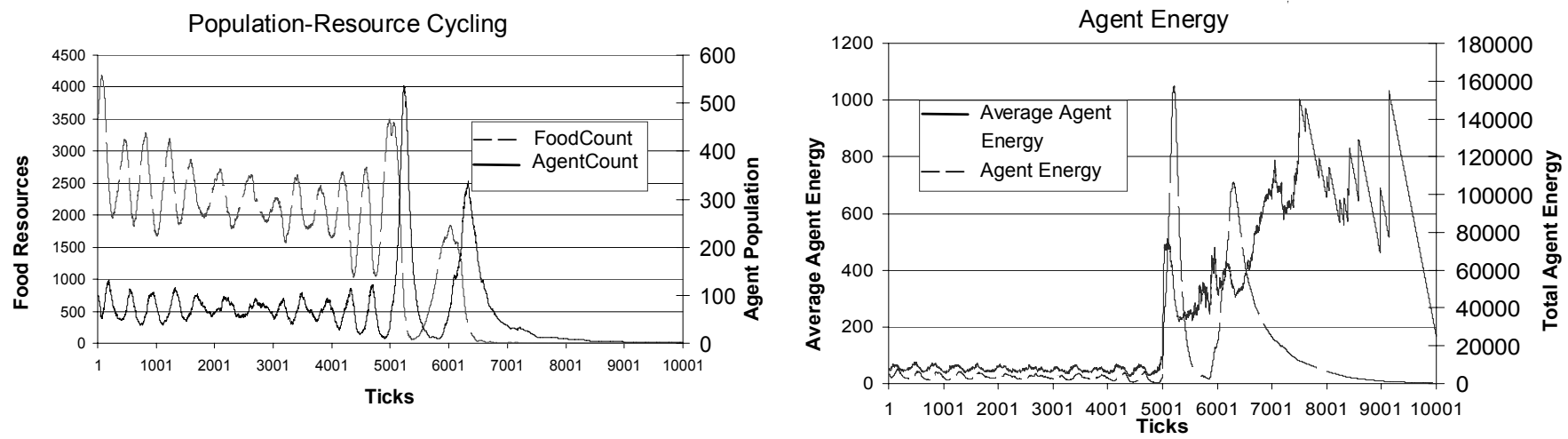

Multi-Phase Run: Food Energy = 10, Raised to 50

Figure 4. Comparing single and multiphase runs with changes in food energy parameter. 
this strategy maintains the health of the agents and a balance between agents and resources. However, when this strategy is applied to an increase in energy availability, the system favours agents that consume more, leading to the eventual elimination of the resource base and the agent population. This latter result suggests one reason why advances in agriculture, specifically increasing the yield or quality of the crops, require additional efforts at pest control. More generally, this result corresponds with other research on the nature of surprise in ecological systems (Bass et al., 1998; Hansell and Bass, 1998; Hansell et al., 1997) that suggests how ecosystems can rapidly jump from one state to another.

An interesting question is why the agents and resources did not become extinct in the single-phase run. It is likely that the dominant strategy that emerges when Food Energy is initially set to 50 is different than the strategy that emerges when Food Energy is set to 10. In the single-phase run with Food Grow at 10, the agent strategy is to consume more and maintain a higher agent count. As the food count is relatively high, the consumption does not wipe out the resource base. When Food Grow is set to 50, the agent strategy is to consume less, as the food count is much lower, and as seen in the multiphase run, too much eating leads to more reproduction which would wipe out the resource base. Changing Food Grow from 10 to 50 in the multiphase run did not allow for this second group of agents to emerge, thus the population wiped out the food base.

The links between resources, population, adaptation and surprise indicate the potential for using COBWEB to gain insight into specific ecological problems. The following section discusses how COBWEB was used to simulate the dynamics of invasive species and the results of those experiments. The simulations of invasiveness build on the more general analysis in this section, suggesting a change in resources, particularly the energy available to the agents, is a critical parameter as is the ability for extinction as agents take advantage of an environmental change.

\section{Simulating Invasive Species}

\subsection{Introduction: Characteristics and Explanations of Invasion}

As a further illustration of how COBWEB can be used in ecological simulation, simulations were developed and experiments were conducted with the baseline simulation to test several hypotheses that have been put forward to explain the success of invading species. The invasiveness of non-indigenous species has increasingly been recognized as one of the major worldwide environmental issues (Miller, 2002). Although the gradual migration of new species into a community can be an important aspect in increasing resilience to change, it can also lead to the dominance of the new species at the expense of the other species in the local community. COBWEB is potentially well suited for simulating the dynamics of invasion as the establishment and spread of new species is dependent on both genetic and evolutionary processes (Sakai et al., 2001), both of which are inherent in COBWEB. In addition, COBWEB appears to be well suited to simulating competition between different types of agents and for experiments with different levels of resources and threshold values.

There are three distinct phases to a successful invasion (Sakai et al., 2001):

1. Introduction of a new species into a new habitat;

2. Colonization and establishment; and

3. Subsequent dispersal and secondary spread into other habitats.

The crucial predictor of success is the attainment of a threshold population at which the invading species can rapidly grow and spread (NRC, 2002). The lag time between the colonization and rapid population growth and expansion into new habitats is an indication of how well the species has adapted to the new environment. Lag times are expected as many introduced species may have initial genetic constraints that would limit their success in the new habitat. Lack of success is often due to a small number of colonists and hence, a population that lacks the genetic diversity of its parent population (Groves and Burdon, 1986). Reduced genetic diversity can result in inbreeding, which in turn limits growth, and the ability to evolve the novel traits required in the new environment (Sakai et al., 2001).

There are other traits that have been linked with successful invasions, although no one trait can serve as a simple predictor of success. Groves and Burdon (1986) have summarized some of these traits, identified in previous studies:

1. High reproductive capacity;

2. High reproductive activity;

3. Rapid growth to sexual maturity;

4. Rapid adaptation to environmental stress (large genetic variation);

5. High tolerance to environmental heterogeneity (plasticity);

6. Significant dispersal ability (range expansion);

7. Ability to out-compete native species.

The Lotka-Volterra competition equation suggests that there are four results (equilibrium solutions to the equation) from an invasion (Shigesada and Kawasaki, 1997):

1. The native species survives; the invader dies out;

2. The invading species survives; and the native species is wiped out;

3. Both species coexist;

4. Stochastic survival of either the native or invading species, depending on initial conditions.

Small populations are prone to stochastic extinction due to chance variation in the survival and reproductive rates of a population, environmental variation and genetic stochasticity including founder effects, genetic bottlenecks and genetic drift (NRC, 2002). Studies of insect infestations suggest that only an estimated $10 \%$ of all non-indigenous species that are 
introduced into a new range become established (NRC, 2002).

There are three explanations of invasion related to the resource base: the Disturbance Hypothesis, the Richness Hypothesis and the Gradient Hypothesis. The Disturbance Hypothesis suggests that changes to the availability of local resources create opportunities for invading species. The Richness Hypothesis suggests that communities high in biodiversity are less prone to invasion, and the Gradient Hypothesis suggests that communities are susceptible to invasion across a gradient, i.e. temperature and rainfall. Studies of resourcebased hypotheses suggest that when the available resources are fully utilized by the resident species, it is more difficult for an invasive species to become established in the new habitat (Shigesada and Kawasaki, 1997).

\subsection{Simulating Invasive Species with COBWEB}

COBWEB is well suited to simulate the first two phases of an invasion and test the contribution of genetic diversity, several life traits (Sections 7.4 to 7.5) and the effect changing resource availability, particularly the Plant and the Disturbance Hypotheses (Sections 7.6 to 7.7). Simulations to test the life traits and the two hypotheses on the success of invasions can be developed with the parameters in Tables 5 and 6. Table 5 indicates the links between COBWEB parameters and the traits of invasive species noted above. Table 6 links COBWEB parameters to favourable environmental characteristics. Sections 7.3 and 7.4 report the results of two control experiments to rule at time of invasion as a means to successful invasion. All simulations involve two species competing for the same resources. As invasion success was defined as the ability of invaders to displace the native species in the habitat, every parameter in Tables 5 and 6 is adjusted to promote invasion. Those changes that result in success are tested further to examine any characteristics of invasion success.

\subsection{Control Experiments}

Experiments were conducted using the default conditions described above to assess the ability of the system to deal with the introduction of agents that have the same characteristics as the native species and to rule out invasion time as a factor of success without any other changes. When the new agents were introduced at the start of the simulation, the dominant species was determined fairly quickly. There were no instances of coexistence, but both the native and the invading agents had an equal probability of success. This is due to the random nature in which the genetics are dispersed to each agent at the initialization of the program. It is typical for one species to receive a favourable strategy or more favourable strategies than another species and out-compete that other species.

In the second set of experiments, new agents, 10 to 200 new individuals, were introduced after 3000 time steps into a native population of approximately 70 individuals. Although there were some cases of coexistence, eventually the native population was able to resist the invaders having the same traits. This was most likely due to the time that the native species had to adapt to the environment. By the time the invaders were introduced, the first set of agents were already well adapted to the variability in the resource base and were able to out compete the invading agents that were not given 3000 time steps to adapt to the environment. When the invaders were introduced at 1000 time steps, to limit the time available to the native agents to adapt to the environmental variability, the native agents were still able to resist the invaders. Thus, without any other changes to the agents or the environment, a new group of agents could not succeed if they were introduced as an invading species.

Table 5. Key Life History Traits and Their Simulation by COBWEB

\begin{tabular}{|c|c|}
\hline Traits & Key COBWEB Parameters \\
\hline $\begin{array}{l}\text { High sexual and asexual } \\
\text { reproductive capacity }\end{array}$ & Breed energy \\
\hline High reproductive activity & $\begin{array}{l}\text { Sexual reproduction probability } \\
\text { Asexual reproduction probability }\end{array}$ \\
\hline $\begin{array}{l}\text { High tolerance to } \\
\text { environmental heterogeneity }\end{array}$ & $\begin{array}{l}\text { Famine (food deplete; deplete } \\
\text { interval) } \\
\text { Food rate } \\
\text { Food grow }\end{array}$ \\
\hline $\begin{array}{l}\text { Significant dispersal ability } \\
\text { (range expansion) }\end{array}$ & Step energy \\
\hline Genetic variability & $\begin{array}{l}\text { Mutation rate } \\
\text { Number of agents }\end{array}$ \\
\hline $\begin{array}{l}\text { Ability to out-compete native } \\
\text { species }\end{array}$ & $\begin{array}{l}\text { Food energy } \\
\text { Step energy } \\
\text { Initial energy } \\
\text { Communication \& memory }\end{array}$ \\
\hline
\end{tabular}

Table 6. Key Environmental Effects and Their Simulation by COBWEB

\begin{tabular}{ll}
\hline $\begin{array}{l}\text { Favourable Environmental } \\
\text { Characteristics }\end{array}$ & Key COBWEB Parameters \\
\hline Repeated introductions & Introduction of new agents \\
$\begin{array}{l}\text { Non-resource based } \\
\text { Environmental disturbance } \\
\text { (effects of pollution) }\end{array}$ & Reproduction probability \\
$\begin{array}{l}\text { Environmental disturbance } \\
- \text { Disturbance hypothesis } \\
\text { (natural hazard) }\end{array}$ & $\begin{array}{l}\text { Famine (food deplete; deplete } \\
\text { interval) } \\
\text { Elimination of resources }\end{array}$ \\
$\begin{array}{l}\text { Environmental disturbance } \\
\text { (shift in environmental } \\
\text { resources) }\end{array}$ & Food grow \\
\hline
\end{tabular}




\subsection{Life History Traits and Invasion Success}

Simulations were conducted with varying numbers of invaders to test the characteristics of highly invasive species detailed in Section 7.1 (Sakai et al., 2001). Invading species were provided with a high initial energy, high reproductive capacity and a higher mutation rate to determine whether these parameters (Table 5), adjusted independently, would enable a species to overcome the native species. Increasing these parameters independently did not lead to a successful invasion. The ability for an invader to out-compete the native species was tested by adjusting the invader's ability to obtain energy from existing resources (increase food energy) and the invader's energy expenditure for movement (reduce Step Energy). Repeated simulations were conducted where Food Energy was doubled and Step Energy was decreased to 1, independently, to provide more opportunity for the introduced agents to out-compete the initial, native species. The invading species were more successful, but only consistently successful with a large number of invaders. In other words, the invaders succeeded in every experiment when they were able to out-compete the native species and invaded in numbers large enough to assume a sufficient strategic diversity (i.e. genetic variety), increasing the chances that some invaders would have successful strategies.

\subsection{Repeat Invasions}

The previous experiments suggested the importance of genetic diversity, represented in COBWEB by different strategies encoded in the genetic algorithms, for an invading species to succeed in a new environment. This was further tested with repeat introductions of a new group of agents to overcome limited genetic diversity in the initial invading population. Two runs were attempted with multiple introductions 25 and 50 agents, every 250 time steps after the initial 3000 time steps for a duration of 10,000 time steps. Neither experiment led to a successful invasion suggesting either that limited genetic diversity is not the principle hindrance to the successful establishment of an invasive species or the requisite genetic diversity can only be attained with larger numbers of invaders.

\subsection{Multiple Food Resource}

The previous experiments suggested that the energy available from the environment was an important factor in determining the success of the invasion was further tested using two resources with different levels of energy. The experiments were set up to exploit the differential ability between the invaders and the native species to extract energy from these resources. Initially, the simulation was tested with two species and two resources providing the same food energy, as a control, to test for any biases in the simulation. As in the previous control experiments, the invasion was not successful. The invaders were then given increased food energy from both resources, and then from only one resource. In both cases, the invasion was successful. However, when the energy available to invaders was increased for one type of resource, but lowered for the other, keeping the total available energy in balance, the invasion was not successful.

\subsection{The Disturbance Hypothesis}

The Disturbance Hypothesis is the leading explanation for the establishment of an invasive species. As an illustration of how this might be tested, COBWEB was used to simulate the natural hazard disturbance with two types of changes in the habitat. The first change tests whether invasion is more likely to be successful if additional resources are made available to the new species. Using COBWEB, the food growth rate was increased with the introduction of a new species. In all cases, the native species was able to adapt to the change in resource availability and out-compete the invading species.

Periodic disturbances were simulated using the famine variables. This was tested with a periodic famine at the start of the invasion and with a periodic famine at every 250 time steps, at the start of the simulation, before the invasion. In only one case, with the introduction of 75 new agents, was the invasion successful. The success was short term as the invaders died after 1000 time steps, during one of the periodic famines.

\subsection{Discussion}

COBWEB has been shown to be a useful tool for investigating those factors that lead to successful invasions, particularly for the first two phases of an invasion. The results of the application of COBWEB to invasive species reproduced the four results Lotka-Volterra competition equation:

1. Invasive species dies, the native species survives, and the invasion is unsuccessful (7.3 and 7.4);

2. Invasive species survives, the native species dies and the invasion is successful (7.4 and 7.6);

3. Both species coexist (7.3 to 7.5 and 7.7);

4. Stochastic survival of one or the other species (7.3, the first control experiment).

In addition, some of the simulations led to the extinction of both species where

1. Invasive species survives, the native species dies, but the invasion is not successful as other factors lead to the extinction of the invader (7.4 and 7.7);

2. Native species survives, and the invasion is unsuccessful, but other factors lead to the extinction of the native species (7.4).

This is a new result is not expected from the Lotka-Volterra competition equation, which only deals with situations when two species compete. These new results occur when one species cannot adapt to the environment and might have been expected based on the results of the experiments conducted in section 6 where an increase in food energy led to an extinction of the agents. The extinction of both species occurred primarily when food energy was increased, which can create a positive feedback on population growth that is unsustainable. 
The experiments conducted with COBWEB supported the importance of genetic variability, the ability of the invaders to out-compete the native species, and the amount of energy available in multiple resources. The lack of invasion success corresponds to earlier cited research suggesting the difficulty in establishing a non-indigenous species. The other traits may still be important explanations of the successful invasions, but these traits were only tested independently, ignoring possible interactions that might emerge if multiple traits were tested in the same experiment. It is also possible to allow both the invaders and the indigenous species to go through a period of adaptation to their respective environments by adding a solid divider into the grid and using the software's manual adjustments to eliminate all but two types of agents, one on each side of the barrier. Even though this would reduce the genetic variability of the invaders, the surviving population would have had opportunity to develop some very resilient strategies and might be better able to take advantage of some of these traits.

The Plant Formation Hypothesis compared the baseline environment, with one resource, to a landscape with two resources with an increase in total available energy to both the indigenous and the invading species. Although these experiments support the results in Section 7.4 for food energy, unlike those experiments, the invasion was always successful with more than one resource as long as the total energy available from the resource base exceeded that in the baseline. This result suggests that even with multiple resources for the invaders, there are still thresholds for available energy that the landscape must provide for a successful invasion.

The Disturbance Hypothesis was also tested in a very simple manner, which favoured the indigenous species. Disturbances should be simulated within the context of creative disturbance, i.e., as events that free up energy for new organisms (Holling, 1985; Hansell and Bass, 1998). This means that disturbances should be simulated in environments with two resources, perhaps growing at different rates favouring the food for the indigenous species, and the disturbance should only affect this resource, freeing up space for the other resource. This type of experiment would behave as a closer representation of the Holling Cycle that will provide more opportunity for a new species to colonize and dominate the environment.

The sudden extinction of one species that was described in Section 6 has been observed in other modelling studies (e.g. Hansell at al., 1997), but the occasional extinction of both species is a result that has not been observed in previous modelling research on invasive species, indicating the ability of COBWEB to generate new results that are consistent with emerging trends in systems ecology. As in Section 6, the extinction of both species was always preceded by large fluctuations in population and resources, which is consistent with earlier work and with the presence of multiple attractors in non-linear models (Hansell et al., 1997) and with earlier research on COBWEB (Bass et al., 2002).

The instances where both species coexisted lasted as long as 5000 time steps, although it was not part of the long-term equilibrium solution, suggests the emergence of semi-stable attractors (Bass et al., 1998; Hansell et al., 1997). Semi-stable attractors are characteristic of some complex systems, but are a relatively recent discovery in ecological models (Byers and Hansell, 1996). These attractors are characterized by the appearance of long-term stability and a sudden change of state to another attractor. The semi-stable attractors have only been formally identified for a system of non-linear differential equations or an iterative functions system and are considered difficult to identify in situ. This behaviour in COBWEB lends credence to the possibility that they can emerge as a result of the actions of individual agents. COBWEB provides more opportunity to investigate these attractors, their emergence and the implications for long-term prediction and management.

\section{Other Ecological Applications}

Because of COBWEB's inherent flexibility, the program can be used to provide insight into many avenues of research by providing a range of experimental tests of different hypotheses. The following are some possibilities for potential research areas in ecology. In some of the areas, further modifications will have to be made to the program to facilitate the exploration of these issues. Some of these modifications will be supported in the next version or can be added directly by the user.

\subsection{Environmental Change \& Population Adaptation}

Humans have extensively altered the global environment, causing severe habitat fragmentation, resource depletion, and the disruption of species interactions. These changes to the ecosystem alter competition, mutualisms and other trophic interactions that frequently lead to greater disruptions among other species, often with strong, unpredictable ecosystem effects (Byers and Hansell, 1996). COBWEB can be adapted to test different hypotheses on the effects of disrupting ecosystem functions, test different characteristics of species at risk, explore different changes that precede the collapse of a system and assess the effects of the extinction of one species on the rest of the system. COBWEB is well suited for representing habitat fragmentation as the user can place stones in any part of the grid to create distinct barriers or obstacles to create a type of isolation. COBWEB also has the potential to simulate anthropogenic effects that spread over an area through time, e.g. gradual urban encroachment, desertification, or other climate change, by modeling the responses of a system to spreading resource depletion or increases in the cost of mobility.

\subsection{Population Adaptation to Spatial Heterogeneity}

All environments possess patches of resource richness and gradients of climatic conditions. The spatial heterogeneity 
of a species' environment has been implicated in the maintenance of genetic diversity and the creation of new species. Furthermore, shifts in the patterns of geographic variation are expected to alter with the global climate change in the next several decades (Holling, 1986). COBWEB could help provide evidence for mechanisms that enable geographic speciation events to take place, clarifying the role of spatial heterogeneity in the maintenance and creation of increased diversity. It could enable a closer study of step clines and their maintenance in the environment and reveal unpredictable changes resulting from spatial rather than temporal changes in the environment. In this application, genetic diversity could be monitored to provide a direct measurement of selection pressures.

\subsection{Infectious Disease \& Determinants of Public Health}

COBWEB could be used in the simulation of vector-borne illness and those diseases transmitted between individuals in the population with some modification to the software. Susceptible, infectious, and resistant individuals in the population would need to be identified and represented in the program. For example, infectious individuals would have to be programmed to spread disease to susceptible individuals. The numbers of each would have to be tracked and new variables like energy cost of fighting infection and duration of illness would have to be incorporated into a new system. For modeling vector-borne diseases, disease vectors would have to be created with the ability to disseminate the disease to the host population.

COBWEB could also be used to explore the relationship between climate change and public health. Not many efforts have been made to model the complexities of environmental health and epidemiology. It is well established that climate is an important determinant of the spatial and temporal distribution of vectors and pathogens. In theory, a change in climate would be expected to cause changes in the geographical range, seasonality, and in the incidence rate of infectious disease. The incidence of mosquito-borne diseases, including malaria, dengue, and viral encephalitides, are among those diseases most sensitive to climate (Patz et al., 1996). Human migration from the projected increase in climate variability could indirectly contribute to disease transmission. It is possible that COBWEB could be modified to model this type of complex interrelationship between humans and their environment. Understanding the linkages between the climatological and ecological change as determinants of disease emergence and redistribution could facilitate development of better preventive strategies.

\section{Conclusions}

The COBWEB simulation model was developed to simulate adaptation to variable and changing environments. It utilizes artificial intelligence applications to replicate decision-making by individuals within a system of agents. The agents' strategies are genetic, but are modifiable through new information derived from the environment or from communication with other agents. COBWEB can simulate the complex behaviour of systems to generate insights that could be generalized for a range of biological and socio-economic systems and is particularly effective in exploring the consequences of environmental change.

The versatility of COBWEB allows it to be used for applications to a wide a variety of ecological problems. An example was provided for invasive species. The results of the simulations supported previous research on the resistance of native species to most invasions, reproduced the results of other types of models and reproduced some of the newer results, such as semi-stability, that are new avenues of research in systems ecology. In addition, the discussion of the results opened up new avenues for research with COBWEB.

COBWEB has several advantages over the standard mathematical approaches due to its ability to simulate the adaptability of populations to variability and change and to simulate the features of complex systems without developing a set of non-linear differential equations. As yet, no other strictly mathematical model has accounted for the capacity of organisms to adapt to changes in the resource base. Furthermore, no other model has accounted for interaction between changes to the resource base and the agent populations while the invasion is in progress. Thus, COBWEB simulations may offer a better representation of invasion than other mathematical approaches due to the ability to capture environment-invader, environment-native species and invader-native species interactions.

The next version of COBWEB will allow the user more flexibility in developing the environment and will test agents that utilize neural nets instead of genetic algorithms. All versions of COBWEB are open source and available from the authors. The software requires a Pentium IV, the most recent Java Runtime Environment and will run under WINDOWS, UNIX and Linux operating systems.

Acknowledgments. The authors would like to acknowledge the contributions of Kenny Chng, Jin Kang, James Martens, Eric Morgen, Pragati Sinha, Rebecca Snell, Nick Toller, Kaleem Ullah, Yana Vasserman and Ruta Wad to this research and the support of the Research Opportunity Program, the Mentorship Program and the Centre for Environment at the University of Toronto.

\section{References}

Bass, B., Byers, R.T. and Lister, N.M. (1998). Integrating research on ecohydrology and land use change with land use management. Hydrol. Process., 12, 2217-2233.

Bass, B., Hill, J. and Suh, N. (2002). Simulating adaptation to environmental change: Complexity and Organized Behaviour Within Environmental Bounds (COBWEB). J. CASYS, 13, 21-33.

Byers, R.E. and Hansell, R.I.C. (1996). Implications of semi-stable attractors for ecological modelling. Ecol. Model., 89, 59-65.

Board on Agriculture and Natural Resources (BANR), Board on Life Sciences (BLS) (2002). Predicting Invasions of Nonindigenous 
Plants and Plant Pests, Washington.

Groves, R.H. and Burton, J.J. (1986). Ecology of Biological Invasions, Australian Academy of Science, Cambridge University Press, Canberra.

Hansell, R.I.C. and Bass, B. (1998). Holling's figure-eight model: A technical reevaluation in relation to climate change and biodiversity. J. Environ. Manage., 49, 157-168.

Hansell, R.I.C., Craine, I.T. and Byers, R.E. (1997). Predicting change in non-linear systems. J. Environ. Manage., 46, 175-190.

Hofstadter, D.R. (1985). Metamagical Themas: Questing for the Essence of Mind and Pattern, Basic Books, NY.

Holling, C.S. (1986). The resilience of terrestrial ecosystems: local surprise and global change, in Clark, W.C. and Munn, R.E. (Eds.), Sustainable Development of the Biosphere, IIASA, Cambridge University Press, pp. 292-317.

Miller, T.E., Kneitel, J.M. and Burns, J.H. (2002). Effect of community structure on invasion success and rate. Ecol., 83(4), 898-905.

Müller, F. and Fath, B. (1998). The physical basis of ecological goal functions-fundamentals, problems and questions, in F. Muller (Ed.), Eco-targets, Goal Functions and Orientors, SpringerVerlag, Berlin-Heidelberg, pp. 15-18.
National Research Council (NRC), Board on Agriculture and Natural Resources (BANR), Board on Life Sciences (BLS), (2002). Predicting Invasions of Noningigenous Plants and Plant Pests: Washington.

Olson, R.L. and Sequeira, R.A. (1995). Emergent computation and the modeling and management of ecological systems. Comput. Electron. Agric., 12, 183-209.

Patz, J.A., Epstein, P.R., Burke, T.A. and Balbus, J.M. (1996). Global climate change and emerging infectious diseases. J. Am. Med. Assoc., 275(3), 217-223.

Scheffer, M. (1990). Multiplicity of stable states in freshwater systems. Hydrobiol., 200/201, 475-486.

Sakai, A.K., Allendorf, F.W., Holt, J.S., Molofsky, J., With, K.A., Baughman, S., Cabin, R.J., Cohen, J.E., Ellstrand, N.C., McCauley, D.E., O’Neil, P., Parker, I.M., Thompson, J.N. and Weller, S.G. (2001). The population biology of invasive species. Annu. Rev. Ecol. Syst., 32, 305-322.

Severinghaus, J.P. and Brook, E.J. (1999). Abrupt climate change at the end of the last glacial period inferred from trapped air in polar ice. Sci., 286(5441), 930-934.

Shigesada, N. and Kawasaki, K. (1997). Biological Invasions: Theory and Practice, Oxford University Press, NY. 